\title{
MOLECULAR-LINE OBSERVATIONS OF THE REMNANT AGB ENVELOPES AROUND PLANETARY NEBULAE
}

\author{
R. SAHAI \\ Chalmers University of Technology, 41296 Gothenburg, Sweden \\ and \\ A. WOOTTEN \\ National Radio Astronomy Observatory, Charlottesville, VA 22903, USA \\ and \\ R.E.S. CLEGG \\ Royal Greenwich Observatory, Madingley Road, Cambridge CB3 OEZ, UK
}

\begin{abstract}
We present recent results from a "search and mapping" program of molecular line emission (mainly $\mathrm{CO}$ ) from remnant $\mathrm{AGB}$ envelopes around planetary nebulae (PNe), using the SEST (La Silla, Chile). New detections in CO J=2-1 include NGC2899 $(0.02 \mathrm{~K})$, NGC6369 $(0.14 \mathrm{~K})$ \& NGC7009 $(0.08 \mathrm{~K})$. In many of the detected PNe, notably NGC3132, IC4406, NGC6302, M1-16, and CPD-56 ${ }^{\circ} 8032$, the molecular envelopes contain 2 kinematically distinct outflows. Mapping of the strongest of these shows (1) that the fast (e.g. $\mathrm{V}_{\text {exp }} \gtrsim 40-60 \mathrm{~km} \mathrm{~s}^{-1}$ in NGC3132, IC4406) outflows have bipolar spatial structure, and (2) there exists an equatorial density enhancement in the slower, more massive $[\dot{M}$ $\left(\mathrm{M}_{\odot} \mathrm{yr}^{-1}\right)>510^{-6}$ (NGC3132), >2 10 $0^{-5}$ (IC4406)] outflows, which presumably collimates the fast outflow (e.g. Sahai et al. 1990, A \& A, 234, L1; Sahai et al. 1992, A \& A, 251, 560). The fractional $\mathrm{CO}$ abundance in the envelope, $\mathrm{f}(\mathrm{CO})$, is probably rather low $\left(<10^{-4}\right)$, as a result of photodissociation by the stellar and interstellar $U V$ radiation [e.g. $f(C O) \leqslant 10^{-5}$ in IC4406]. $\mathrm{HCN}, \mathrm{HCO}^{+}$, and ${ }^{13} \mathrm{CO}$ have also been detected in several PNe, and sensitive upper limits set on $\mathrm{CS}, \mathrm{C}^{18} \mathrm{O}, \& \mathrm{C}^{17} \mathrm{O}$ (in M1-16), and SO (in NGC3132). Some results are tabulated below, and calculations to estimate the molecular masses, mass-loss rates and molecular abundances are in progress.
\end{abstract}

\begin{tabular}{|c|c|c|c|c|c|c|}
\hline Mol./Line & Data & $\mathrm{V}_{\mathrm{c}}^{\mathrm{a}}$ & $\Delta v^{b}$ & $\Delta \mathrm{V}_{\mathrm{hi}}{ }^{\mathrm{c}}$ & $\mathrm{T}_{\mathrm{mb}}(\mathrm{K})$ & Comments \\
\hline \multicolumn{3}{|c|}{ M: Map, S: Spectrum } & \multicolumn{2}{|c|}{ M1-16 } & & \\
\hline $\mathrm{CO} \mathrm{J}=2-1$ & M & 50.5 & 44 & 60 & 1.8 & $\mathrm{HVW}^{\mathrm{d}}, \mathrm{B}^{\mathrm{e}}(\mathrm{NW}-\mathrm{SE})^{\mathrm{f}}$, opt. thick \\
\hline $\mathrm{CO} \mathrm{J}=1-0$ & $\mathrm{~S}$ & 50.5 & 43 & 60 & 0.43 & HVW, opt. thick \\
\hline${ }^{13} \mathrm{CO} \mathrm{J}=1-0$ & $\mathrm{~S}$ & 50.2 & 38 & - & 0.14 & {$\left[{ }^{12} \mathrm{C}\right] /\left[{ }^{13} \mathrm{C}\right]<10, \dot{\mathrm{M}}>10^{-4} \mathrm{M}_{\mathrm{yr}} \mathrm{r}^{-1}$ ? } \\
\hline $\mathrm{HCN} \mathrm{J}=1-0$ & $\mathrm{~S}$ & $\approx 50$ & $\approx 43$ & $160 ?$ & $0.025 \mathrm{~V}$ & Very high-vel $(-20$ to 140$)$ emission? \\
\hline $\mathrm{HCO}^{+} \mathrm{J}=1-0$ & $\mathrm{~S}$ & $\approx 50$ & $\approx 43$ & $\approx 70$ & 0.043 & \\
\hline $\mathrm{N}_{2} \mathrm{H}^{+} \mathrm{J}=1-0$ & $\mathrm{~S}$ & $\approx 47$ & $\approx 31$ & - & 0.03 & Double-peaked line?(marginal det.) \\
\hline \multicolumn{6}{|c|}{ CPD- $56^{\circ} 8032$} & \\
\hline $\mathrm{CO} \mathrm{J}=2-1$ & M & -57 & 50 & 186 & 0.58 & HVW, B (NWW-SEE), opt. thick \\
\hline $\mathrm{CO} \mathrm{J}=1-0$ & $\mathrm{~S}$ & -57 & 50 & 120 & 0.13 & HVW \\
\hline${ }^{13} \mathrm{CO} \mathrm{J}=1-0$ & $\mathrm{~S}$ & $\approx-57$ & $\approx 50$ & - & 0.04 & {$\left[{ }^{12} \mathrm{C}\right] /\left[{ }^{13} \mathrm{C}\right]<10, \dot{\mathrm{M}}>10^{-4} \mathrm{M}_{\odot \mathrm{yr}} \mathrm{r}^{-1} ?$} \\
\hline $\mathrm{HCO}^{+} \mathrm{J}=1-0$ & $\mathrm{~S}$ & $\approx-57$ & $\approx 50$ & - & $\approx 0.035$ & \\
\hline \multicolumn{6}{|c|}{ NGC3132 } & \\
\hline $\mathrm{CO} \mathrm{J}=2-1$ & M & -25 & 30 & 70 & 0.35 & HVW, B ( $\approx N W-S E)$, opt. thick \\
\hline $\mathrm{CO} \mathrm{J}=1-0$ & $\mathrm{~S}$ & -25 & 30 & 70 & 0.24 & HVW, opt. thick \\
\hline${ }^{13} \mathrm{CO} \mathrm{J}=1-0$ & $\mathrm{~S}$ & $\approx-25$ & $\approx 18$ & - & 0.035 & \\
\hline \multicolumn{5}{|c|}{ NGC6302 } & 0.045 & Broad profile \\
\hline $\mathrm{CO} \mathrm{J}=2-1$ & M & -36 & 60 & 110 & 0.9 & HVW, B Expanding Lobes $(\approx \mathrm{E}-\mathrm{W})$ \\
\hline $\mathrm{CO} \mathrm{J}=1-0$ & M & $\approx-30$ & $\approx 55$ & $\approx 100$ & $\approx 0.25$ & (contamination from I.S. emission) \\
\hline $\mathrm{HCO}^{+} \mathrm{J}=1-0$ & $\mathrm{~S}$ & $\approx-36$ & $\approx 30$ & - & 0.025 & \\
\hline $\mathrm{HCN} \mathrm{J}=1-0$ & $\mathrm{~S}$ & $\approx-40$ & $\approx 40$ & -- & 0.025 & \\
\hline
\end{tabular}

a- VLSR $\left(\mathrm{km} \mathrm{s}^{-1}\right) ; \mathrm{b} \& \mathrm{c}-\mathrm{Full}$-widths at zero intensity $\left(\mathrm{km} \mathrm{s}^{-1}\right)$ of main \& high-velocity components in central spectrum; $d$ - High-vel. wings in spectrum; e (f)- Bipolar (axis) 